\title{
MULTI-LEVEL NON-RIGID IMAGE REGISTRATION USING GRAPH-CUTS
}

\author{
Ronald W. K. So and Albert C. S. Chung \\ Lo Kwee-Seong Medical Image Analysis Laboratory, \\ Department of Computer Science and Engineering, \\ The Hong Kong University of Science and Technology, Hong Kong \\ \{csswk,achung\}@cse.ust.hk
}

\begin{abstract}
Non-rigid image registration is widely used in medical image analysis and image processing. It remains a challenging research problem due to its smoothness requirement and high degree of freedoms in the deformation process. In [1], a method is proposed to solve non-rigid image registration via graph-cuts algorithm by modeling the registration process as a discrete labeling problem. A displacement label (vector) is assigned to each pixel in the source image to indicate the corresponding position in the floating image. The whole system is then optimized by using the graph-cuts algorithm via alphaexpansions [2]. As the initial point is not required for the graph-cuts algorithm, the method proposed by [1] is a singlelevel registration. In this paper, rather single-level, we enable multi-level non-rigid image registration using graph-cuts by passing the deformation field of the current resolution level to the successive finer one. By applying the proposed multilevel registration method, the number of labels used in each level is greatly reduced due to lower image resolution being used in coarser levels. Therefore, the speed of the registration process is improved. We compare our results with the original single-level version, DEMONS and FFD. It is found that our method improves the speed of non-rigid image registration by $50 \%$ and meanwhile maintains similar robustness and registration accuracy with the single-level version.
\end{abstract}

Index Terms - Non-rigid image registration, multi-level graph cuts.

\section{INTRODUCTION}

Non-rigid Image registration is a challenging problem because of the requirement of smoothness and high degree of freedoms, and is widely used in medical image analysis, e.g. [3] shows the extensive use of non-rigid image registration. The goal of image registration is to find an optimal transformation $T^{*}$ such that each pixel of the source image $I$ can be spatially matched, which is measured by a dissimilarity function $C(I, T(J))$, to a pixel of the floating image $J$. In formula, that is:

$$
T^{*}=\arg \min _{T} C(I, T(J)) .
$$

Image registration can be broadly classified into two main categories, rigid and non-rigid, according to the nature of transformation $T$. Transformation in rigid image registration is constrained to rigid transformation, which is a global transformation consists of a rotation and followed by a translation, whereas no common model is used for transformation in nonrigid image registration. Without the penalty term, all pixels in non-rigid image registration are allowed to transform freely. Thus, penalty term is added as a constrain to keep the transformation smooth. This is the main reason that nonrigid image registration is challenging as we should strike a balance between high degree of freedom and smoothness of transformation in the image deformation process.

As the transformation $T$ in Eq. 1 allows any points in $J$ to be mapped to any points in $I$ regardless of the smoothness of the deformation field. Here, a penalty term $S(T)$ should be included to the Eq. 1 in order to pose smoothness constrain on $T$,

$$
T^{*}=\arg \min _{T}(C(I, T(J))+\lambda S(T)),
$$

where $\lambda$ is a positive constant that governs the strength of the penalty.

Finding the optimal transformation for non-rigid image registration can be modeled as a labeling problem. In [1], it is modeled as a labeling problem, which is optimized by using graph-cuts via alpha-expansions [2]. Eq. 2 is reformulated as,

$$
D^{*}=\arg \min _{D}(C(I(X), J(X+D))+\lambda S(D)),
$$

where $D$ is a deformation vector field representing the transformation $T, I$ and $J$ are the source image and the floating image respectively, $X$ is continuous spatial domain of both images, $C$ denotes dissimilarity function and $S$ is a smoothness function. Integrated absolute difference is used as dissimilarity function $C$ and the first derivative terms are used as the smoothness function $S$. As $X$ is discretized into pixels, Eq. 3 becomes

$$
\begin{array}{r}
D^{*}=\arg \min _{D}\left(\sum_{x \in X}\|I(x)-J(x+D(x))\|\right. \\
\left.+\lambda \sum_{(x, y) \in N}\|D(x)-D(y)\|\right),
\end{array}
$$


where $(x, y) \in N$ iff $x$ and $y$ are adjacent pixels. This formula in an energy form has been already described in $[4,2]$ which can be solved by using graph-cuts. The next step is to convert the optimization into a labeling problem. A discretized window $W=\{0, \pm s, \pm 2 s, \ldots, \pm w s\}^{d}$ is applied, which is obtained by discretize the continuous dimension- $d$ region $[-w s, w s]^{d}$, such that $D(x) \in W$, where $s$ is the sampling period. Thus, the labeling problem can be solved by using graph-cuts and alpha-expansions [2]. (Please refer to [1] for details.)

In [1], a single-level non-rigid image registration method was proposed. The main reason is that graph-cuts can minimize the energy function and give global minimum or a local minimum in a strong sense without initialization. Therefore, single-level registration is enough as optimization in any level is independent to other levels. In this paper, we propose a multi-level non-rigid image registration by passing the deformation vector field across resolution levels. With multi-level registration, the number of labels used in each registration level is greatly reduced due to the fact that lower image resolution levels are used and the deformation vector field will be scaled to meet the image resolution in the next finer level. The experimental results show that the speed of the image registration process is remarkably improved, and similar robustness and registration accuracy are maintained as compared with the single-level image registration. The next section describes the way to pass the deformation vector field across resolution levels in order to achieve multi-level non-rigid image registration using graph-cuts. Section 3 shows the experimental results of the proposed method and compares the results with the single-level version, DEMONS [5] and Free Form Deformations Based Method (FFD) [6]. Finally, section 4 summarizes the results.

\section{METHODOLOGY}

Let $n \in[0,1, \ldots, L]$ be the registration levels, where $L+1$ is total number of resolution levels and 0 is the coarsest level, and $D_{n}$ be the deformation vector field in registration level $n$. Thus, Eq. 4 is refined as:

$$
\begin{array}{r}
D_{n}^{*}=\arg \min _{D_{n}}\left(\sum_{x \in X_{n}}\left\|I_{n}(x)-J_{n}\left(x+D_{n}(x)\right)\right\|\right. \\
\left.+\lambda \sum_{(x, y) \in N}\left\|D_{n}(x)-D_{n}(y)\right\|\right),
\end{array}
$$

where $I_{n}$ and $J_{n}$ are the source image and the floating image used in level $n$ respectively, $X_{n}$ is continuous spatial domain of both $I_{n}$ and $J_{n}$, and $D_{n}(x)$ is the deformation vector assigned to pixel $x \in X_{n}$.

We use Gaussian pyramid (course-to-fine) for multi-level registration and images are successively refined by decreasing the spacing by a scale factor $r$. Starting from the coarsest level, each registration level obtains an optimized solution
$D_{n}^{*}$ for the labeling problem by using graph-cuts via alphaexpansions [2]. The next step is to pass $D_{n}^{*}$ to level $n+1$ and use it to initialize $D_{n+1}$. As the result, the whole process ends in the original level and $D_{L}^{*}$ is the final solution for the whole registration. Since each pixel is assigned a deformation vector, the size of $D_{n}$ depends on the image resolution in level $n$. Due to the difference in image resolution used in different registration levels, the sizes of deformation vector fields in different levels are not the same. Therefore, $D_{n}^{*}$ should be re-scaled to fit $D_{n+1}$ before it is passed to the next resolution level. Empty cells of re-scaled $D_{n}^{*}$ are calculated by averaging deformation vectors of adjacent pixels. The resulting deformation field is used to initialize $D_{n+1}$ by multiplying $r$ to each vector of the resulting deformation field. We can combine and formulate the re-scaling process and initialization process as:

$\forall x \in X_{n+1}$,

$$
D_{n+1}(x)= \begin{cases}r D_{n}^{*}(x / r) & : \text { Case I, } \\ \frac{\sum_{y \in Y} D_{n+1}(y)}{|Y|} & \text { :Case II, }\end{cases}
$$

where $Y$ represents all pixels adjacent to $x$ and $|Y|$ denotes the number of pixels in $Y$. Case I and Case II are conditions representing the re-scaling process and initialization process respectively. They are defined as:

Case I: If all coordinates of $x$ are divisible by $r$,

Case II: Otherwise.

All cells of $D_{n+1}$ which belong to Case I are handled before Case II. We multiply the scale factor $r$ in Case I due to the difference in image resolutions. Images used in level $n, I_{n}$ and $J_{n}$, are down-sampled from images used in level $n+1, I_{n+1}$ and $J_{n+1}$, by the scale factor $r$. Thus, if a pixel $i$ of source image is mapped to pixel $x$ of floating image in level $n$, then, in level $n+1$, the corresponding pixel of source image should be mapped to pixel $r x$ of floating image. Observably, it is equivalent to multiplying the corresponding deformation vector by $r$. In order to keep the smoothness of the deformation field, the missing cells of $D_{n+1}$ are initialized by averaging vectors assigned to its adjacent pixels.

Since down-sampled images are used in lower registration level, the number of labels can be reduced in order to speed up the whole registration process. As the result, the size of the discretized window $W=\{0, \pm s, \pm 2 s, \ldots, \pm w s\}^{d}$, which controls the maximum displacement of deformation vectors, is significantly reduced by the scale factor $r$. Then, the set of labels for optimized solution $D_{n}^{*}$ in each level is obtained from the resulting discretized window. The computation time needed to solve the multi-label problem by using graph-cuts via alpha-expansion [2] mainly depends on the number of labels. As such, reducing number of labels at each level can greatly improve the speed of the registration process. Unlike single-level version of non-rigid image registration using graph-cuts which works in one optimization process, multilevel version divides the registration into several levels. Each 
level $n$ is optimized by graph-cuts and the resulting deformation vector field $D_{n}^{*}$ is passed to the next finer level after re-scaling. Together with the initialization of $D_{n+1}$ and lower resolution images are used in coarser levels, number of labels used in each level can be reduced. Meanwhile, it is valuable to note that labels used in the original level also is reduced as the last coarser level can deal with pixels of far displacement and hence speed up the whole registration process.

\section{EXPERIMENTAL RESULTS}

We followed the experimental settings of [1], i.e., image pairs, which are intensity normalized to be within 0 and 255 , used in all experiments are assumed affinely pre-registered. Moreover, implementations of FFD and DEMONS used in experiments were obtained from ITK [7] where $15 \times 15$ control point grid was used in FFD. Implementation of grph-cuts algorithm are obtained from the source codes provided by Kolmogorov $\&$ Zabih [4]. For single-level non-rigid registration using graph-cuts (denoted as single-level GC later), we used $\lambda=$ $0.05 \times 255$ and $W=\{0, \pm 1, \pm 2, \ldots, \pm 15\}^{2}$. In our method, we used $\lambda=0.05 \times 255, W=\{0, \pm 1, \pm 2, \ldots, \pm 8\}^{2}$ for all levels, scale factor $r=2$ and 4 registration levels. Thus, deformation labels(vectors) assigned to pixels were chosen from $31 \times 31$ window for single-level or $17 \times 17$ window for all levels of multi-level. Source image used in our experiments were obtained from BrainWeb [8], the source image and its segmented images are shown in Fig.1. Five floating images were obtained by applying different artificial deformations (Case A to E) to the source image which are shown in the left column of Fig.2. All experiments were performed on an Intel $2.13 \mathrm{GHz}$ dual-core CPU with $1 \mathrm{~GB}$ RAM.

Columns 2-5 of Fig.2 show the registration results of Case A-E of our method, single-level GC, DEMONS and FFD respectively. It is observed that our method and single-level GC are successful in all cases whereas DEMONS fails in Case C-E and FFD failed in Case B and D. The failures of Case C$\mathrm{E}$ of DEMONS are due to the fact that it allows high-degree of freedom and uses local intensity gradient to optimize the movement of pixels. Therefore, if some area of brain tissue of the source image is mapped to the background of floating image (or reverse), like Case C-E, the optimization process may be trapped in a local minima as some pixels are driven towards wrong directions. Although our method and singlelevel GC also allow high-degree of freedom, they are able to handle such cases. The reason is that graph-cuts assign an label(vector) to each pixel, i.e., movement of pixel, in an alpha-expansion in a global manner.

Unlike our method, single-level GC and DEMONS, FFD use hard constrain on the movement of pixels. Only control points in FFD can be moved freely, and the displacements of all other pixels are calculated from its neighborhood control points via some interpolation functions like B-spline. As this low-degree of freedom, FFD cannot model complicated or high frequency deformation like Case B and D. DEMONS is

\begin{tabular}{|c|cc|c|}
\hline & Our Method & Single-Level & \% Reduced \\
\hline Case A & $526 \mathrm{~s}$ & $1128 \mathrm{~s}$ & $53.37 \%$ \\
Case B & $502 \mathrm{~s}$ & $1121 \mathrm{~s}$ & $55.22 \%$ \\
Case C & $567 \mathrm{~s}$ & $1200 \mathrm{~s}$ & $52.75 \%$ \\
Case D & $569 \mathrm{~s}$ & $1215 \mathrm{~s}$ & $53.17 \%$ \\
Case E & $530 \mathrm{~s}$ & $1237 \mathrm{~s}$ & $57.15 \%$ \\
\hline Average & $538.8 \mathrm{~s}$ & $1180.2 \mathrm{~s}$ & $54.33 \%$ \\
\hline
\end{tabular}

Table 1. Computational times for one registration of 5 different artificial deformation cases.

successful in Case B due to the high degree of freedom. Since high degree of freedom and global manner optimization, our method and single-level GC are successful in all cases.

With the helps of passing the deformation field, our method enables multi-level non-rigid registration, significantly reduces the number of labels used in each registration level, including the original level, such that speeds up the whole registration process. Table 1 lists and compares the computation times of Case A-E of our method and single-level GC. It shows that our method can reduce more than $50 \%$ of the computation time meanwhile maintain similar robustness and registration accuracy with the single-level GC.

\section{CONCLUSION}

In this paper, we have proposed a new method to pass the deformation field across resolution levels in order to enable multi-level non-rigid registration using graph-cuts. Experimental results show that our method inherits high degree of freedom and global optimization from the single-level version, and also is robust to different challenging deformation cases. Meanwhile, our newly proposed multi-level registration method can reduce the number of labels used in each level especially the original level. As such, the registration time can be reduced by $50 \%$. The future research directions include the extension of our approach to 3D and applying more advance metrics, like mutual information, to our method.

\section{REFERENCES}

[1] Tommy W. H. Tang and Albert C. S. Chung, "Non-rigid image registration using graph-cuts," MICCAI 2007, LNCS, vol. 4791, pp. 916-924, 2007.

[2] Y. Boykov, O. Veksler, and R. Zabih, "Fast approximate energy minimization via graph cuts," PAMI, vol. 23 (11), pp. 1222-1239, 2004.

[3] D. Rueckert, "Non-rigid registration: Techniques and applications," in Medical Image Registration, CRC Press, 2001.

[4] V. Kolmogorov and R. Zabih, "What energy functions can be minimized via graph cuts?," PAMI, vol. 26 (2), pp. 147-159, 2005.

[5] J. Thirion, "Image matching as a diffusion process: an analogy with maxwell's demons," MedIA, vol. 2 (3), pp. 243-260, 1998.

[6] D. Rueckert, L. I. Sonoda, and et al, "Non-rigid registration using freeform deformations: Application to breast mr images," TMI, vol. 18 (8), pp. 712-721, 1999.

[7] L. Ibanez, W. Schroeder, and et al, The ITK Software Guide, 1st edn. kitware edition, 2003.

[8] B. Aubert-Broche, A. Evans, and L. Collins, "A new improved version of the realistic digital brain phantom," NeuroImage, vol. 32 (1), pp. 138-145, 2006. 


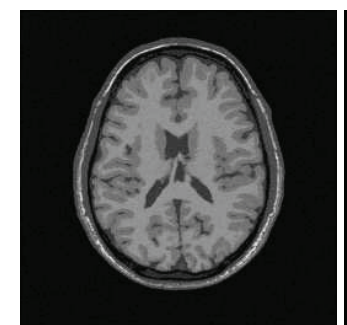

Source Image

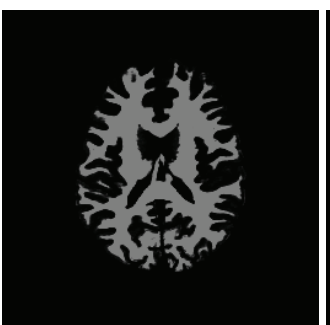

WM

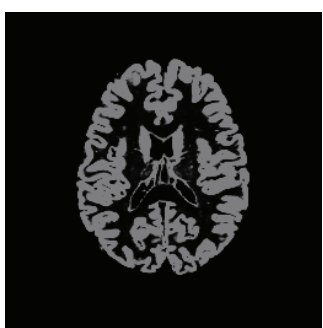

GM

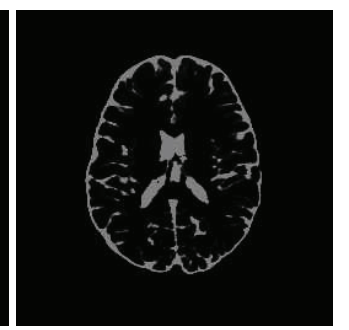

CSF

Fig. 1. Source and its segmented images obtained from BrainWeb used in our experiments.

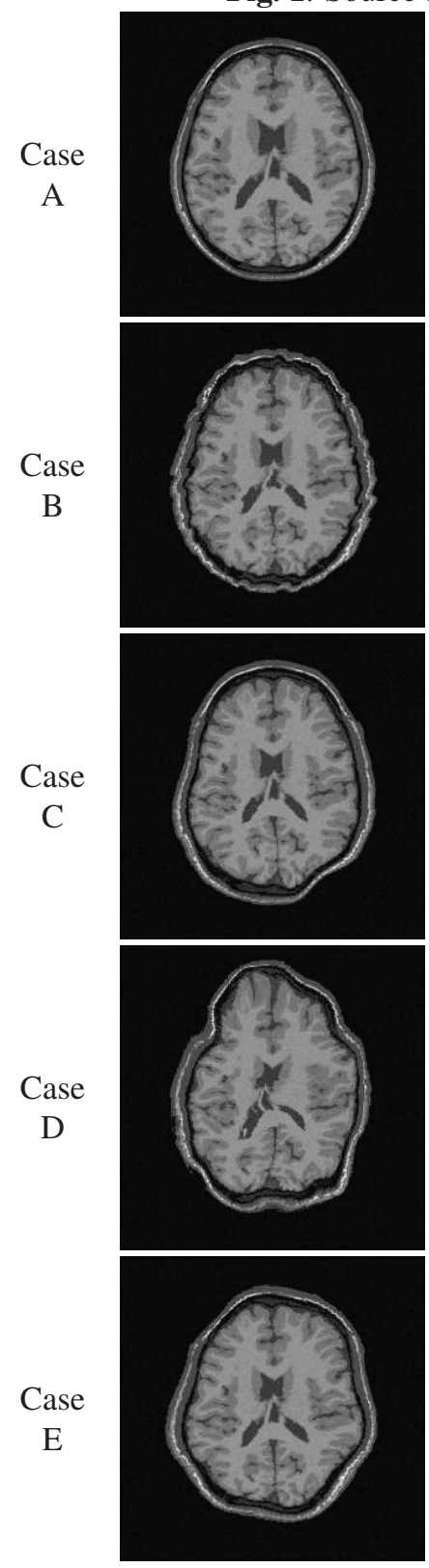

Floating
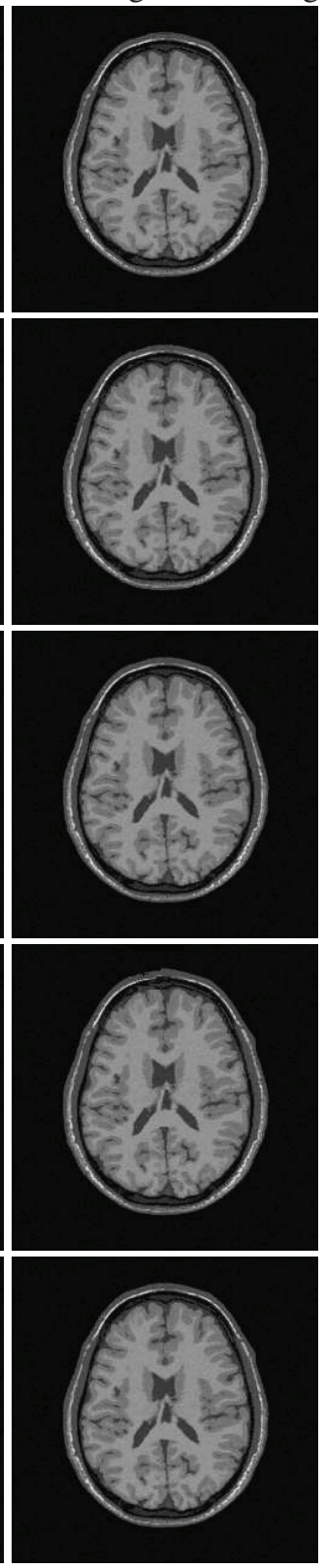

Our Method
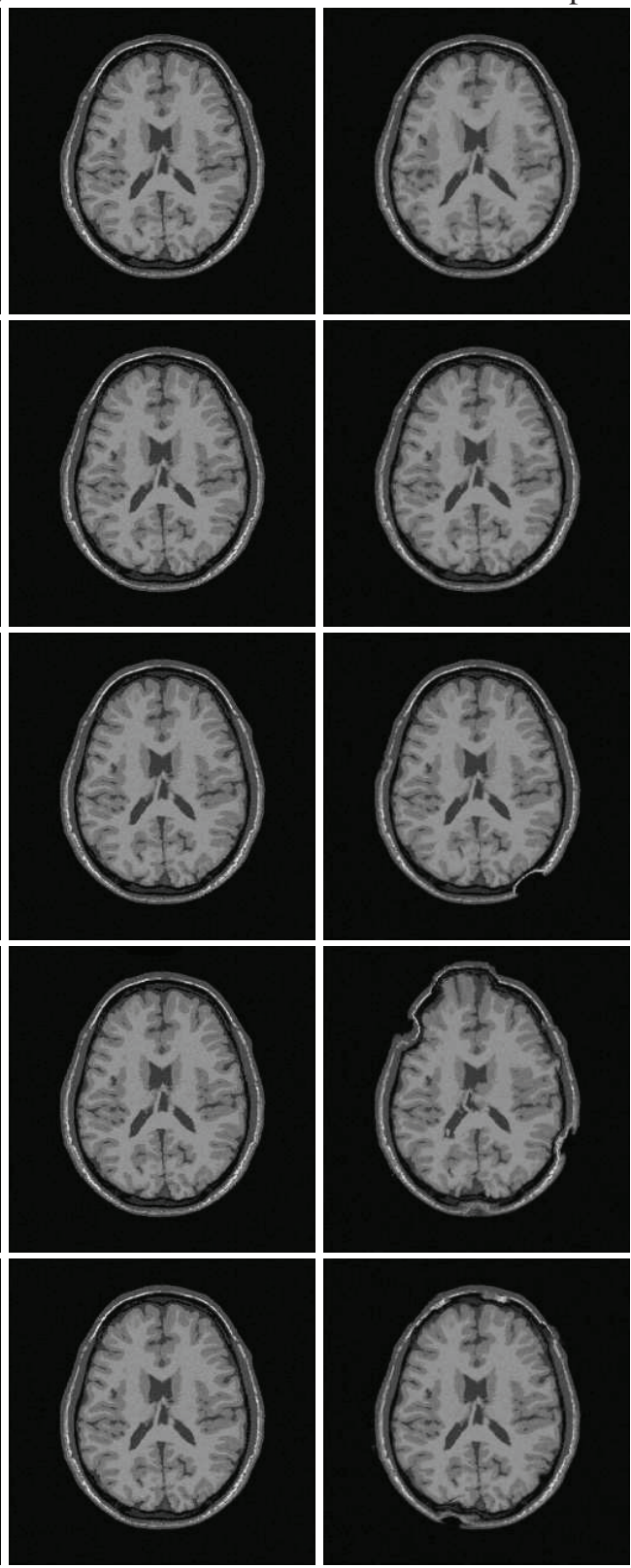

Single-Level
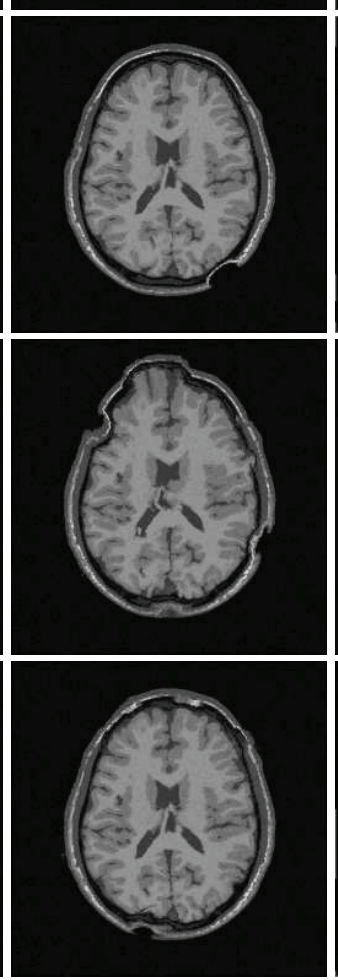

DEMONS
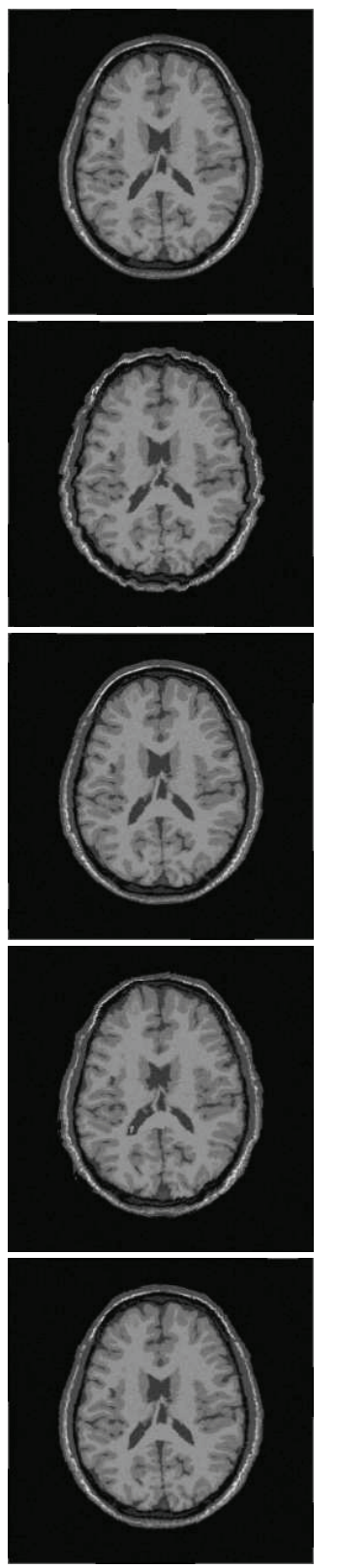

FFD

Fig. 2. Results of 5 different artificial deformation cases. 